\title{
Detection of Power System Faults in the Presence of Linear Loads Using Stockwell Transform
}

\author{
Neha Agarwal ${ }^{1}$, Om Prakash Mahela ${ }^{2}$, Bipul Kumar ${ }^{3}$ \\ ${ }^{I}$ (M.Tech Scholar, Department of Electrical Engineering, RCEW, Jaipur, India) \\ ${ }_{2}^{2}$ (Assistant Engineer, Rajasthan Rajya Vidhyut Prasaran Nigam Ltd., Jodhpur, India) \\ ${ }^{3}$ (Assistant Professor, Department of Electrical Engineering, RCEW, Jaipur, India)
}

\begin{abstract}
This paper presents the detection of power system faults such as Line to ground fault (LG), double line fault $(L L)$, double line to ground fault $(L L G)$ and three-phase fault involving ground $(L L L G)$ in the presence of resistive-inductive load. The Stockwell's transform has been utilized for the detection of these faults. The proposed study has been carried out in the MATLAB/Simulink environment. The faults have been simulated at $6^{\text {th }}$ cycle and results are plotted for 12 cycles. The S-transform based decomposition of voltages for all the three phases as well as current related to all the three phases have been carried out in each case of the study to obtain the S-matrix. The frequency contents and variance curve have been obtained from the S-matrix. These are utilized to detect and discriminate the various types of power system faults from each other. The S-transform based contour and variance curve has been utilized for detection of the faults.
\end{abstract}

Keywords: Double line fault, double line to ground fault, line to ground fault, linear load, three-phase fault, Stockwell Transform.

\section{Introduction}

Transmission lines are the life blood of the power delivery. When a fault is detected, the protective relay must respond quickly to isolate the faulted line to preserve the stability of the rest of the system. Accurate estimates of the fault location are desirable for inspection, maintenance, and repair of the actual fault. Thus, the protective relaying and fault location of transmission lines are very important subjects [1]. The fault diagnosis in power systems includes the detection of the time origin of failures with the identification and location of the event that occurred in these systems. The restoration of faulty equipment must take place in a fast and efficient way, stimulating the research in fault diagnosis systems [2]. In Electrical Power System (EPS) there are some power swings in which currents and voltages waveforms behave as a fault and can result in mis-operation of distance relays [3]. Various signal processing techniques such as wavelet transform (WT), neural networks, Fuzzy logic etc. have been widely used for the detection and classification of the power system faults [4]. In the context of fault diagnosis in electric power systems, a detailed survey to review the intelligent systems application to fault diagnosis in electric power system transmission lines has been reported in [5].

A novel approach for detecting, classifying and locating short-circuit faults in power transmission lines has been reported in [6]. Based on the proposed approach, a hybrid framework consisting of a proposed two stage finite impulse response (FIR) filter, four support vector machines (SVMs), and eleven support vector regressions (SVRs) is implemented in Proteus 6/MATLAB environments. A low cost, fast and reliable microcontroller based protection scheme using wavelet transform and artificial neural network has been proposed by the authors in [7] and its effectiveness has been evaluated in real time. Shaik et al. [8], presented the application of wavelet transforms for the detection, classification and location of faults on transmission lines. In the proposed methodology a Global Positioning System clock has been utilized to synchronize sampling of voltage and current signals at both the ends of the transmission line. The detail coefficients of current signals of both the ends are utilized to calculate fault indices. This paper presents the detection of power system faults such as Line to ground fault (LG), double line fault (LL), double line to ground fault (LLG) and three-phase fault (LLLG) in the presence of resistive-inductive load. The Stockwell's transform has been utilized for the detection of the above mentioned power system faults. The proposed study has been carried out in the MATLAB/Simulink environment.

\section{Proposed Test System}

The single line diagram of test system used for proposed study related to the detection of faults in the presence of resistive-inductive loads is shown in Fig. 1. The conventional generator Gen is connected to the network through the transformer (Trafo-1). The generator generates the power at $120 \mathrm{kV}$ voltage level. The fundamental frequency is taken as $60 \mathrm{~Hz}$. The transmission line TRL is connected in network between the buses $\mathrm{B} 1$ and B2 as shown in the test system. The transformer Trafo-1 steps down the voltage level from $120 \mathrm{kV}$ to 25 $\mathrm{kV}$. The positive and zero sequence impedances of transmission lines are $(0.1153+\mathrm{j} 0.3958) \Omega / \mathrm{km}$ and 
$(0.4130+\mathrm{j} 1.2516) \Omega / \mathrm{km}$ respectively. The length of each transmission line $T R L$ is $25 \mathrm{~km}$. This line has been operated at $25 \mathrm{kV}$ voltage level in the network. The fault is created on the bus B-2 with the help of three-phase fault block in the MATLAB. The resistive-inductive load has the capacity of $10 \mathrm{MW}$ and 3 MVAR reactive load. The technical data of transformer are provided in Table 1.

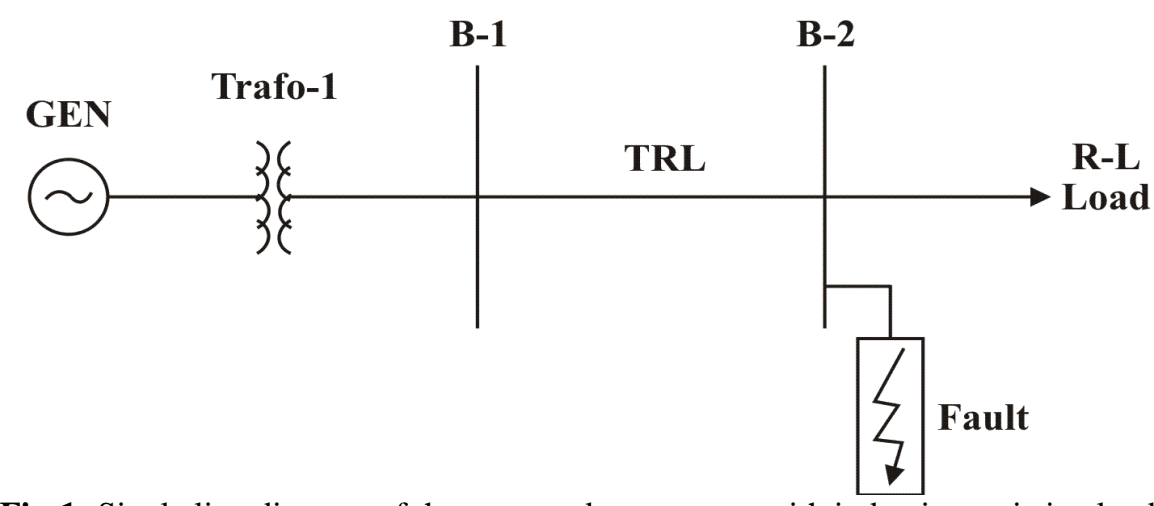

Fig-1: Single line diagram of the proposed test system with inductive resistive load.

Teble-1: Transformer Data

\begin{tabular}{|l|l|l|l|l|l|l|l|}
\hline Transformer & MVA & \multirow{2}{*}{ kV-High } & \multirow{2}{*}{ kV-Low } & \multicolumn{2}{|l|}{ HV winding } & \multicolumn{2}{l|}{ LV winding } \\
\cline { 5 - 8 } & & & & $\mathrm{R}(\mathrm{pu})$ & $\mathrm{L}(\mathrm{pu})$ & $\mathrm{R}(\mathrm{pu})$ & $\mathrm{X}(\mathrm{pu})$ \\
\hline Trafo-1 & 47 & 120 & 25 & 0.002 & 0.08 & 0.002 & 0.08 \\
\hline
\end{tabular}

\section{Proposed Algorithm}

The test system is simulated in MATLAB/Simulink environment with resistive-inductive load. The detection of various types of power system faults such as LG, LL, LLG and LLLG on transmission line has been investigated. The study has been carried out with resistive-inductive load using the test system shown in Fig. 1 . Bus B-1 is selected as the test point and voltage and current signals are captured at this bus. The results have been plotted for 12 cycles. The faults have been initiated at the end of $6^{\text {th }}$ cycle on the bus B-2 of the test system. The captured voltage and current signals have been decomposed using the Stockwell's transform with a sampling frequency of $3.2 \mathrm{kHz}$ and $\mathrm{S}$-matrix is obtained. The $\mathrm{S}$-contour and variance curve obtained from the $\mathrm{S}$ matrix are plotted for each power system fault. These plots are analyzed to detect and discriminate the various types of power system faults.

\section{Simulation Results and Discussion}

This section presents the simulation results related to the detection of power system faults such as Line to ground fault (LG), double line fault (LL), double line to ground fault (LLG) and three-phase fault (LLLG) in the presence of resistive-inductive load using Stockwell Transform.

\subsection{Line to Ground Fault}

The line to ground fault on phase-A has been simulated on the bus B2 of the test system shown in Fig. 1 at $6^{\text {th }}$ cycle from the start of the simulation. The S-transform based decomposition of the voltage and current for all the three phases is carried out and S-matrix is obtained for each data set of the voltage and currents.

The voltage signal of phase-A, S-contour for the voltage of phase-A and variance curve obtained from S-matrix for the voltage of phase-A are shown in Figs. 2 (a), (b), and (c) respectively. It can be observed from the S-contour shown in the Fig. 2 (b) that the high frequency isolated contour is observed which helps in the detection of the line to ground fault on the bus B2 of the test system. From the Fig. 2 (c) it has been observed that the sharp peak appears in the variance curve of the voltage at the time of occurrence of fault which helps in the detection of the fault on the power system network. Magnitude of the variance curve decreases after fault. The plots have also been investigated for the standard deviation obtained from the S-matrix and compared with the respective plot of the variance. It has been observed that the variance plot is more effective as compared to the standard deviation plot. Hence, we have utilized the variance plot. 

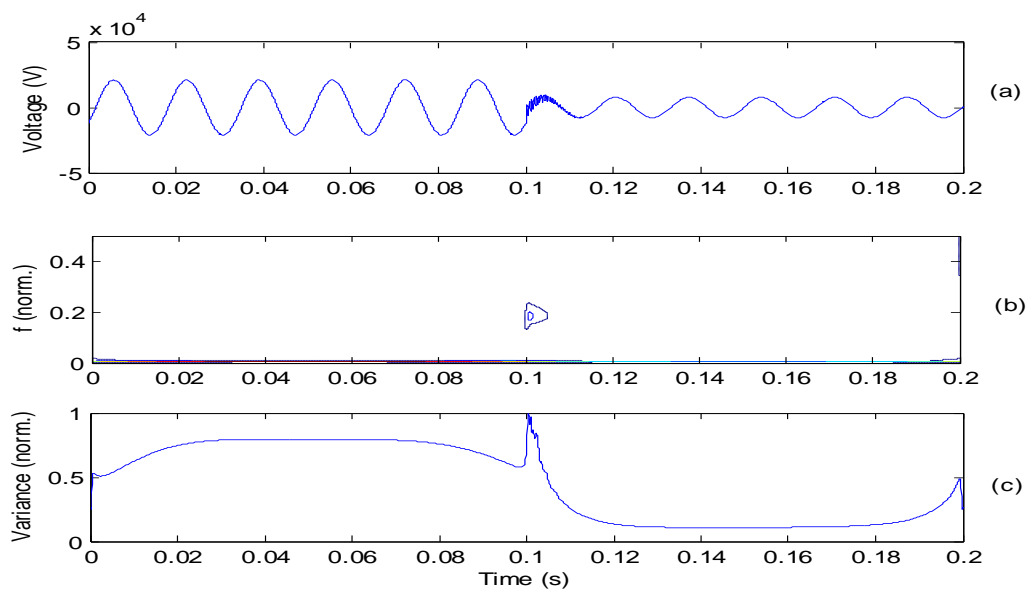

Fig-2: S-transform based plots for the voltage of the phase-A during LG fault on phase-A (a) voltage waveform of phase-A (b) S-contour and (c) variance plot.

The S-transform based decomposition of the voltage of phase-B during the LG fault on the phase-A has been carried out and S-matrix is obtained. The voltage signal of phase-B, S-contour for the voltage of phase-B and variance curve obtained from S-matrix for the voltage of phase-B are shown in Figs. 3 (a), (b), and (c) respectively. It can be observed from the S-contour shown in the Fig. 3 (b) that the high frequency isolated contour is also detected in the phase-B voltage. However, the frequency contents are with low magnitude as compared to the faulty phase i.e. phase-A. From the Fig. 3 (c) it has been observed that the sharp peak also appears in the variance curve of the voltage of phase-B at the time of occurrence of the fault on the phase-A which helps in the detection of the fault on the power system network. However, the magnitude of the peak is very less as compared to corresponding peak for the faulty phase which helps in distinguising the healthy phase from the faulty phase. The magnitude of variance curve increases after faulty event.
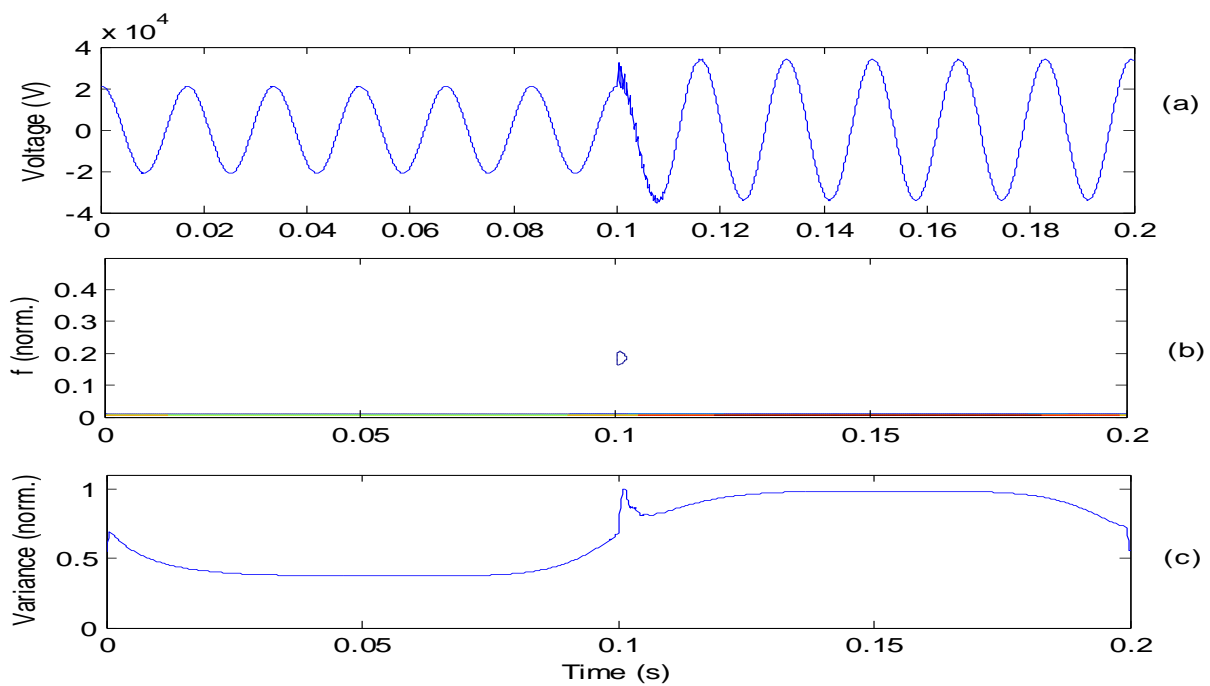

(c)

Fig-3: S-transform based plots for the voltage of phase-B during LG fault on phase-A (a) voltage waveform of phase-B (b) S-contour and (c) variance plot.

The S-transform based decomposition of the current in the phase-A during the LG fault on the phase-A has been carried out and S-matrix is obtained. The current of phase-A, S-contour for the current in the phase-A and variance curve obtained from S-matrix for the current in the phase-A are shown in Figs. 4 (a), (b), and (c) respectively. It can be observed from the S-contour shown in the Fig. 4 (b) that the high frequency isolated contour is not detected in the phase-A current whereas the magnitude of the current is high during the post fault time duration as compared to the pre-fault current. From the Fig. 4 (c) it has been observed that the magnitude of the variance curve also increases after the faulty event indicating the increase in the current during the post fault event. However, the sharp peak does not appears in the variance of the current in phase-A. Hence, the Stransform based decomposition of the current only indicates the related magnitude of the current before and after the fault event. 

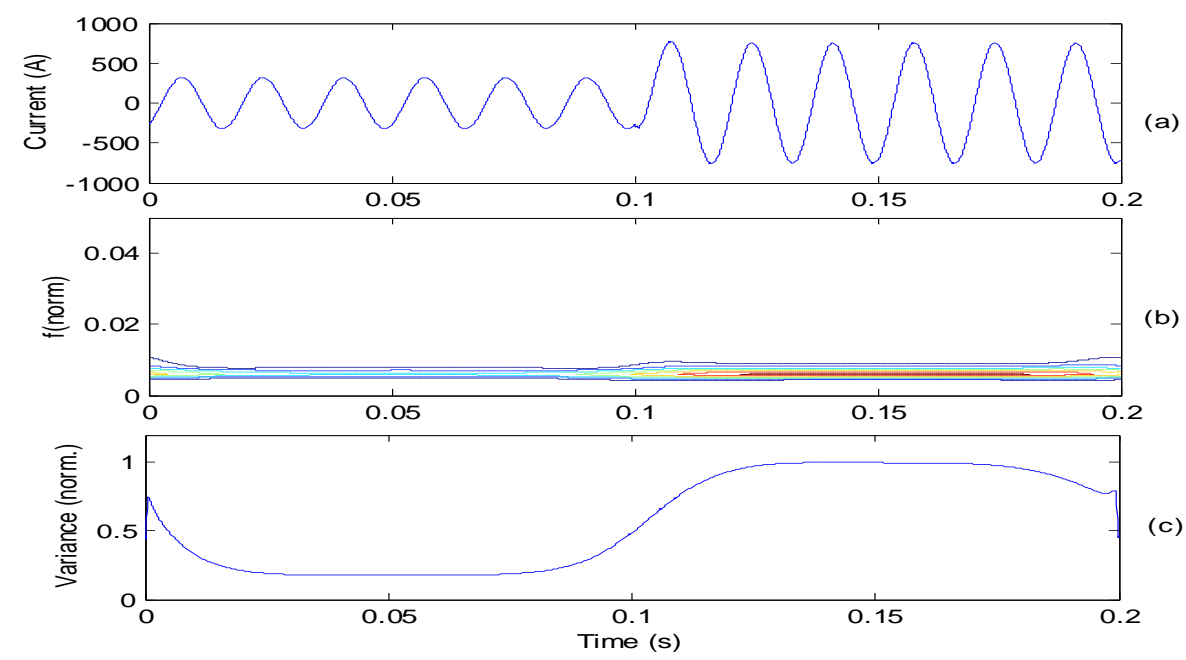

Fig-4: S- transform based plots for the currtent of the phase-A during LG fault on phase-A (a) current waveform of phase-A (b) S-contour and (c) variance plot

The S-transform based decomposition of the current in the phase-B during the LG fault on the phase-A has been carried out and S-matrix is obtained. The current of phase-B, S-contour for the current in the phase-B and variance curve obtained from S-matrix for the current in the phase-B are shown in Figs. 5 (a), (b), and (c) respectively. It can be observed from the S-contour shown in the Fig. 5 (b) that the high frequency isolated contour is not detected in the phase-B current whereas the magnitude of the current is high during the post fault time duration as compared to the pre-fault current. From the Fig. 5 (c) it has been observed that the magnitude of the variance curve also increases after the faulty event. However, the sharp peak does not appears in the variance of the current in phase-A. Hence, the S-transform based decomposition of the current only indicates the related magnitude of the current before and after the faulty event.
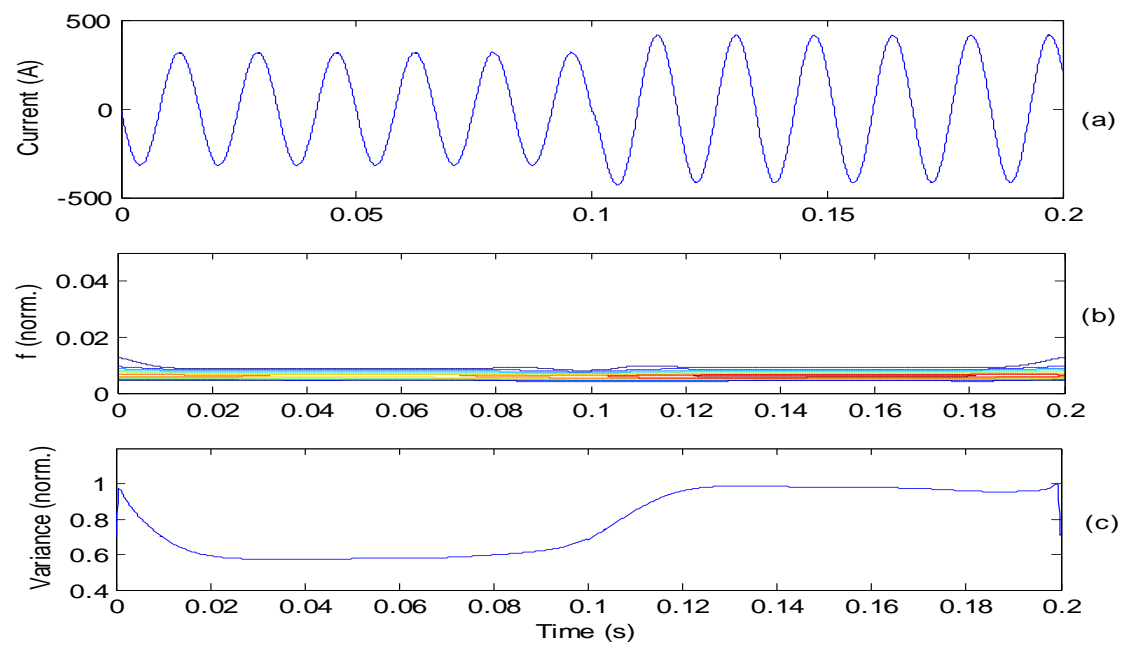

Fig-5: S- transform based plots for the currtent of the phase-B during LG fault on phase-A (a) current waveform of phase-B (b) S-contour and (c) variance plot.

\subsection{Double Line Fault}

The double line or line to line (LL) fault has been simulated on bus B2 of the test system shown in Fig. 1 at $6^{\text {th }}$ cycle from the start of the simulation by short circuiting the phases A \& B. The S-transform based decomposition of the voltage and current for all the three phases is carried out and S-matrix is obtained for each data set of the voltage and currents. The voltage signal of phase-A, S-contour for the voltage of phase-A and variance obtained from S-matrix for the voltage of phase-A during LL fault are shown in Figs. 6 (a), (b), and (c) respectively. It can be observed from the S-contour shown in the Fig. 6 (b) that an abrupt change is observed in the contour which helps in the detection of the line to ground fault on the bus B2 of the test system. From the Fig. 6 (c) it has been observed that the abrupt small magnitude sharp depression appears in the variance of the voltage at the time of occurrence of the fault which helps in the detection of the fault on the power system network. 


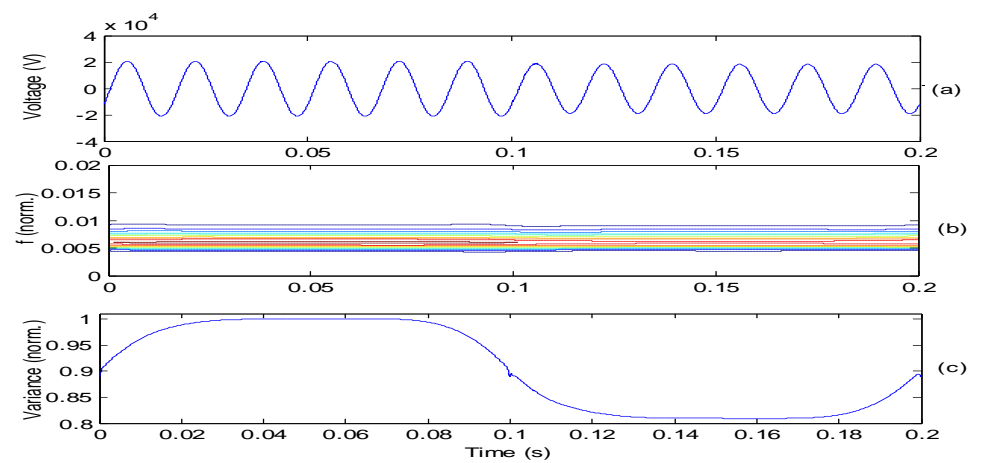

Fig-6: S- transform based plots for the voltage of phase-A during double line fault on phases-A \&B (a) voltage waveform of phase-A (b) S-contour and (c) variance plot.

The S-transform based decomposition of the voltage of phase-C (healthy phase) during the LL fault on the phase-A has been carried out and S-matrix is obtained. The voltage signal of phase-C, S-contour for the voltage of phase-C and variance obtained from S-matrix for the voltage of phase-C are shown in Figs. 7 (a), (b), and (c) respectively. It can be observed from the S-contour shown in the Fig. 7 (b) that significant changes have not been observed in the S-contour related to the phase-C voltage. From the Fig. 7 (c) it has also been observed that the sharp peaks are not observed in the variance of the voltage of phase- $\mathrm{C}$ at the time of occurrence of the LL fault on the phases-A \& B. Hence, during the LL fault changes are observed in the S-transform plots of voltage of the faulty phases and no changes have been observed in the S-transform based plots of the healthy phases.

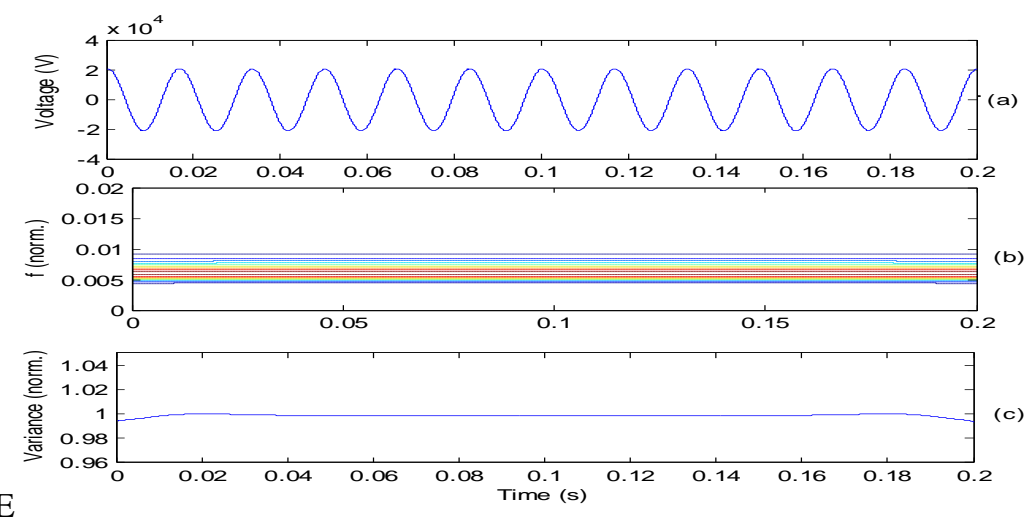

Fig-7: S- transform based plots for the voltage of phase-C during double line fault on phases-A \&B (a) voltage waveform of phase-C (b) S-contour and (c) variance plot.

The S-transform based decomposition of the current in the phase-A during the LL fault on the phasesA \& B has been carried out and S-matrix is obtained. The current of phase-A, S-contour for the current in the phase-A and variance obtained from S-matrix for the current in the phase-A are shown in Figs. 8 (a), (b), and (c) respectively. It can be observed from the S-contour shown in the Fig. 8 (b) that the magnitude of the S-contour increases suddenly at the time of the ocurrence of the fault. From the Fig. 8 (c) it has been observed that the magnitude of the variance curve also increases after the faulty event.

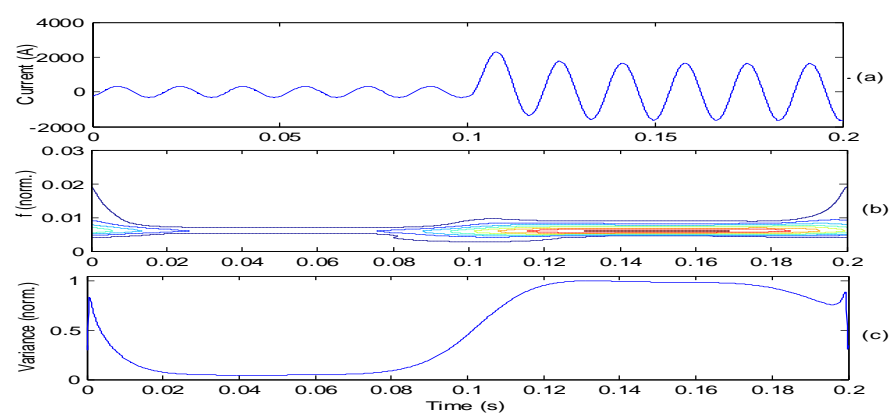

Fig-8: S- transform based plots for the current in phase-A during double line fault on phases-A \&B (a) current waveform of phase-A (b) S-contour and (c) variance plot. 
The S-transform based decomposition of the current in the phase-C during the LL fault on the phases-A $\& \mathrm{~B}$ has been carried out and S-matrix is obtained. The current of phase-C, S-contour for the current in the phase-C and variance obtained from S-matrix for the current in the phase-C are shown in Figs. 9 (a), (b), and (c) respectively. It can be observed from the S-contour shown in the Fig. 9 (b) that the magnitude of the S-contour remains constant. From the Fig. 9 (c) it has been observed that the magnitude of the variance curve also remains constant during the faulty event.

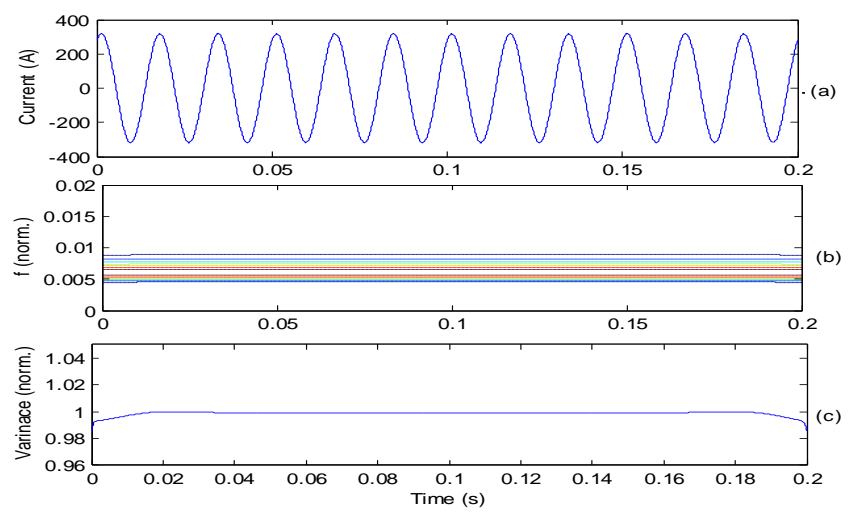

Fig-9: S- transform based plots for the current in phase-C during double line fault on phases-A \&B (a) current waveform of phase-C (b) S-contour and (c) variance plot

\subsection{Double Line to Ground Fault}

The double line to ground (LLG) fault has been simulated on bus B2 of the test system shown in Fig. 1 at $6^{\text {th }}$ cycle from the start of the simulation by connecting the phases A \& B to the ground. The S-transform based decomposition of the voltage and current for all the three phases is carried out and S-matrix is obtained for each data set of the voltage and currents. The voltage signal of phase-A, S-contour for the voltage of phase-A and variance obtained from S-matrix for the voltage of phase-A during LLG fault are shown in Figs. 10 (a), (b), and (c) respectively. It can be observed from the S-contour shown in the Fig. 10 (b) that an isolated contour appears in the S-contour which helps in the detection of the LLG fault on the bus B2 of the test system. The area covered by this contour is large as compared to the area covered by the similar contour with the LG fault. From the Fig. 10 (c) it has been observed that the high magnitude sharp peak appears in the variance of the voltage at the time of occurrence of the fault which helps in the detection of the fault on the power system network.
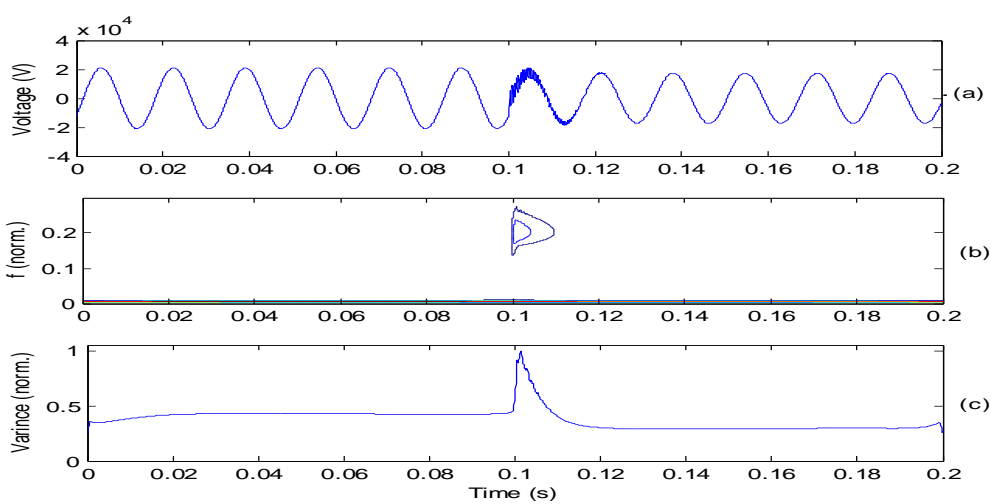

Fig-10: $S$ - transform based plots for the voltage of phase-A during double line to ground fault on phases-A \&B

(a) voltage waveform of phase-A (b) S-contour and (c) variance plot.

The voltage signal of phase-C, S-contour for the voltage of phase-C and variance obtained from Smatrix for the voltage of phase-C during LLG fault on the phases A\&B are shown in Figs. 11 (a), (b), and (c) respectively. It can be observed from the S-contour shown in the Fig. 11 (b) that an isolated contour appears in the S-contour which helps in the detection of the LLG fault on the bus B2 of the test system. The area covered by this contour is large as compared to the area covered by the corresponding contour of the healthy phase with the LLG fault. However, the area of this contour is less as compared to the respective curve of the faulty phase. From the Fig. 11 (c) it has been observed that the high magnitude sharp peak appears in the variance of the voltage at the time of occurrence of the fault which helps in the detection of the fault on the power system network. 


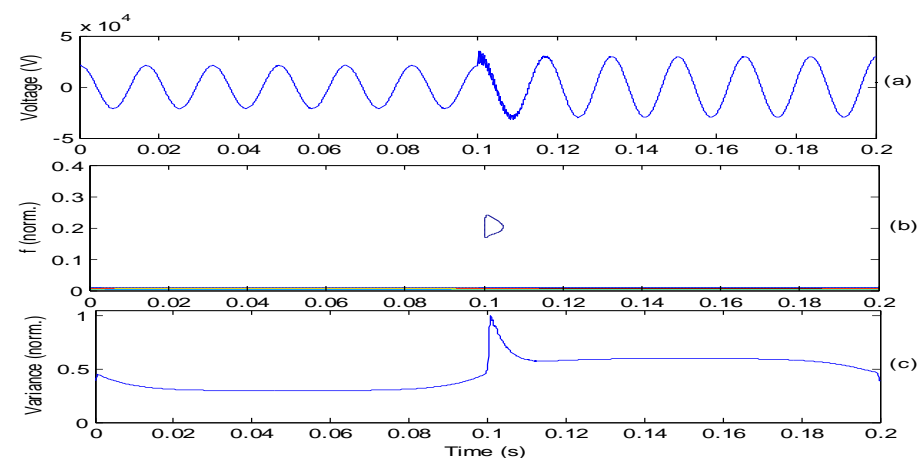

Fig-11: S- transform based plots for the voltage of phase-C during double line to ground fault on phases-A \&B (a) voltage waveform of phase-C (b) S-contour and (c) variance plot.

The S-transform based decomposition of the current in the phase-A during the LLG fault on the phases-A \& B has been carried out and S-matrix is obtained. The current of phase-A, S-contour for the current in the phase-A and variance obtained from S-matrix for the current in the phase-A are shown in Figs. 12 (a), (b), and (c) respectively. It can be observed from the S-contour shown in the Fig. 12 (b) that the magnitude of the Scontour increases suddenly at the time of the ocurrence of the fault. From the Fig. 12 (c) it has been observed that the magnitude of the variance curve also increases after the faulty event.

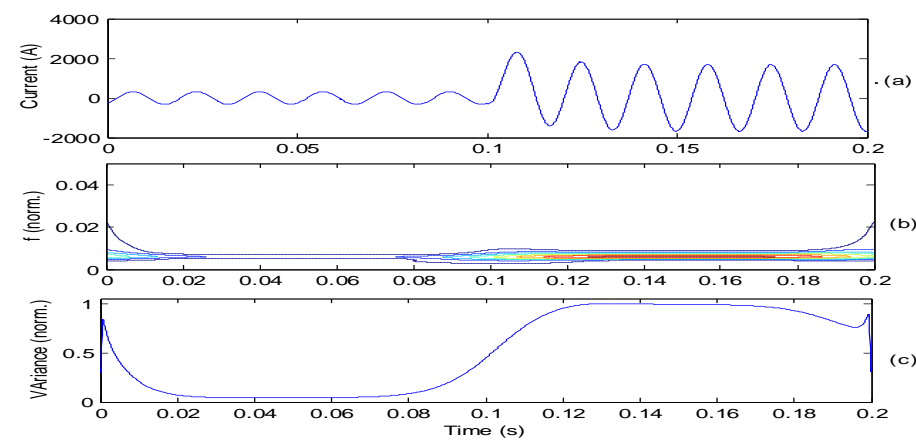

Fig12: S- transform based plots for the current in the phase-A during double line to ground fault on phases-A $\& B$ (a) current waveform of phase-A (b) S-contour and (c) variance plot.

The S-transform based decomposition of the current in the phase-C during the LLG fault on the phasesA \& B has been carried out and S-matrix is obtained. The current of phase-C, S-contour for the current in the phase-C and variance obtained from S-matrix for the current in the phase-C are shown in Figs. 13 (a), (b), and (c) respectively. It can be observed from the S-contour shown in the Fig. 13 (b) that the magnitude of the Scontour increases suddenly at the time of the ocurrence of the fault and then becomes again constant. The difference in the magnitude of the pre-fault and post-fault event is small. The change in the form of bump at the time of fault helps the detection of fault and discrimination of the faulty and healthy phases. From the Fig. 13 (c) it has been observed that the magnitude of the variance curve also increases after the faulty event.

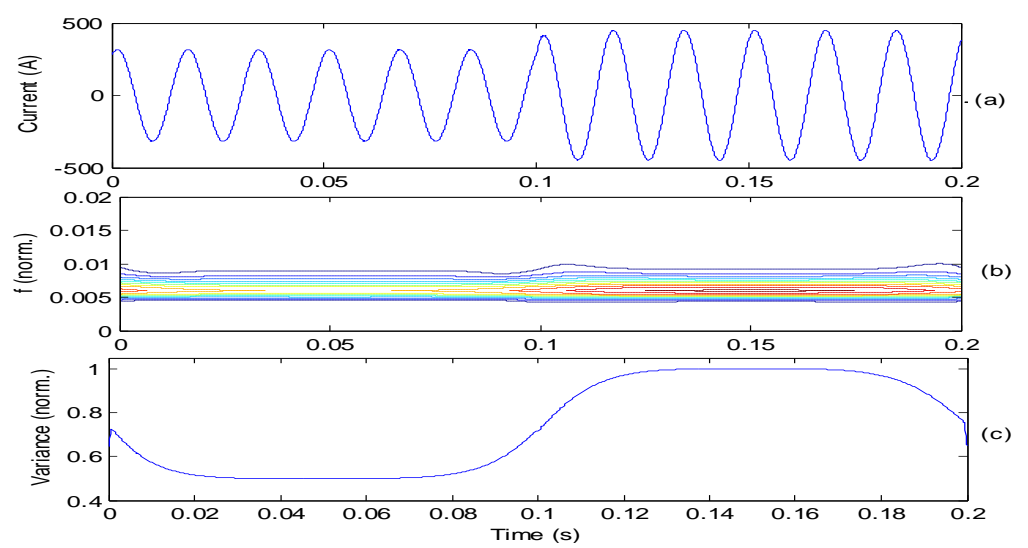

Fig-13: S- transform based plots for the current in the phase-C during double line to ground fault on phases-A $\& B$ (a) current waveform of phase-C (b) S-contour and (c) variance plot 


\subsection{Three-Phase Fault Involving Ground}

The three-phase fault involving ground (LLLG) has been simulated on bus B2 of the test system shown in Fig. 1 at $6^{\text {th }}$ cycle from the start of the simulation by connecting all the three phases to ground. The Stransform based decomposition of the voltage and current for all the three phases is carried out and S-matrix is obtained for each data set of the voltage and currents.

The voltage signal of phase-A, S-contour for the voltage of phase-A and variance obtained from Smatrix for the voltage of phase-A during LLLG fault are shown in Figs. 14 (a), (b), and (c) respectively. It can be observed from the S-contour shown in the Fig. 14 (b) that voltage magnitude decreases with a small bump which helps in the detection of the LLLG fault on the bus B2 of the test system. From the Fig. 14 (c) it has been observed that the high magnitude sharp peak appears in the variance of the voltage at the time of occurrence of the fault which helps in the detection of the fault on the power system network. The rising slope of the peak is very high as compared to the other types of the faults.

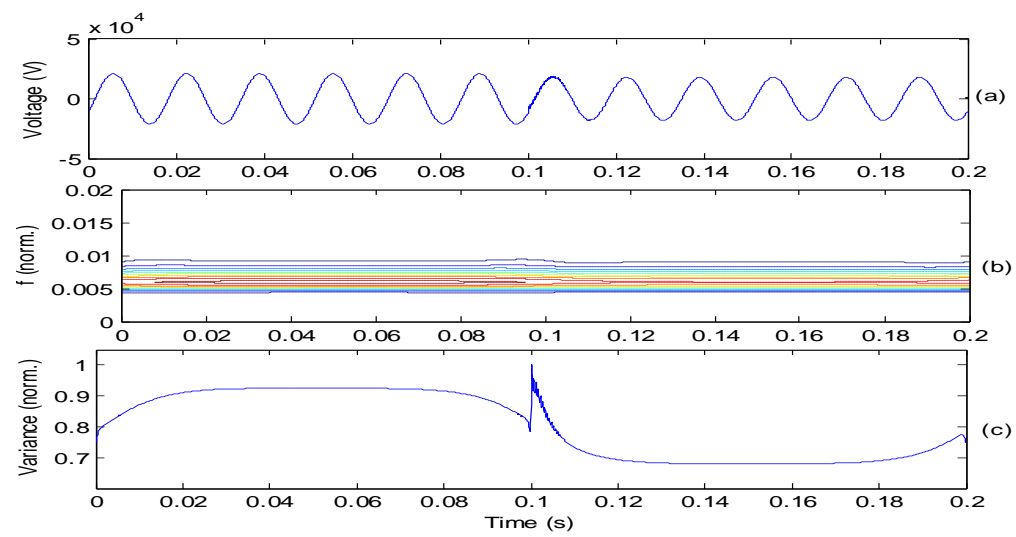

Fig-14: S- transform based plots for the voltage of the phase-A during three-phase fault involving ground (a) voltage waveform of phase-A (b) S-contour and (c) variance plot.

The S-transform based decomposition of the current in the phase-A during the LLLG fault has been carried out and S-matrix is obtained. The current of phase-A, S-contour for the current in the phase-A and variance obtained from S-matrix for the current in the phase-A are shown in Figs. 15 (a), (b), and (c) respectively. It can be observed from the S-contour shown in the Fig. 15 (b) that the magnitude of the S-contour increases suddenly at the time of the ocurrence of the fault with bump at the time of occurrence of the fault. From the Fig. 15 (c) it has been observed that the magnitude of the variance curve increases after the faulty event.

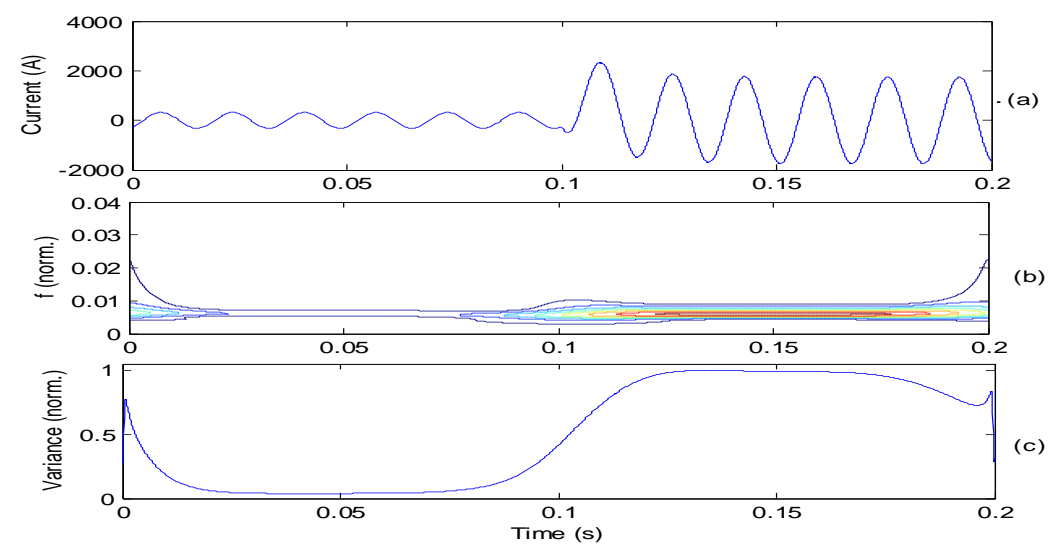

Fig. 15: S- transform based plots for the current in the phase-A during three-phase fault involving ground (a) current waveform of phase-A (b) S-contour and (c) variance plot

\section{Conclusion}

A study of detection of the various types of transmission line faults such as LG, LL, LLG and LLLG in the presence of resistive-inductive loads has been carried out using the Stockwell transform. The S-contour and variance curve obtained from the S-matrix related voltage and current signals have been utilized to detect and discriminate the various types of faults from each other. It has been concluded from the proposed study that in 
case of the unsymmetrical faults involving ground isolated contour representing high frequency contents appear in the S-contour of the voltage at the time of fault occurrence. The area of this contour is high with the faulty phase as compared to the healthy phase. The isolated contour is not observed with the double line fault without involving the ground and symmetrical fault. The high magnitude peaks have been observed in the variance curve of the voltage at the time of occurrence of the fault. The nature of slope of this curve and relative magnitude of this curve helps in the detection of the various types of faults in the presence of the resistiveinductive load as well as in the discrimination of the various types of faults and faulty phase from the healthy phases. The abrupt changes in the S-contour of the current and relative magnitude of S-contour and variance curve of the current during the pre-fault and post fault conditions will help in the detection and discrimination of the various types of faults as well as the faulty and healthy phases.

\section{References}

[1]. Joe-Air Jiang, Ching-Shan Chen, and Chih-Wen Liu, "A New Protection Scheme for Fault Detection, Direction Discrimination, Classification, and Location in Transmission Lines," IEEE Transactions on the Power Delivery, Vol. 18, No. 1, January 2003.

[2]. V.H. Ferreira, R. Zanghi, M.Z. Fortes, G.G. Sotelo, R.B.M. Silva, J.C.S. Souza, C.H.C. Guimarães, and S. Gomes Jr, “A survey on intelligent system application to fault diagnosis in electric power system transmission lines," Electric Power Systems Research, Vol. 136, pp. 135-153, 2016

[3]. A. P. Morais, G. Cardoso Júnior, L. Mariotto, and G. Marchesan, "Fault location scheme for multi-terminal transmission lines using unsynchronized measurements," International Journal of Electrical Power and Energy Systems, Vol. 78, pp. 277-284, 2016.

[4]. Ch. Durga Prasad, N. Srinivasu, "Fault Detection in Transmission Lines using Instantaneous Power with ED based Fault Index," Proceedia Technology, Vol. 21, pp. 132 - 138, 2015.

[5]. V.H. Ferreira, R. Zanghi, M.Z. Fortes, G.G. Sotelo, R.B.M. Silva, J.C.S. Souza, C.H.C. Guimarães, S. Gomes Jr.Fsjjglds, “A survey on intelligent system application to fault diagnosis in electric power system transmission lines," Electric Power Systems Research, Vol. 136, pp. 135-153, 2016

[6]. Hassan Fathabadi, "Novel filter based ANN approach for short-circuit faults detection, classification and location in power transmission lines," International Journal of Electrical Power and Energy Systems, Vol. 74, pp. 374-383, 2016.

[7]. Ebha Koley, Raunak Kumar, Subhojit Ghosh, "Low cost microcontroller based fault detector, classifier, zone identifier and locator for transmission lines using wavelet transform and artificial neural network: A hardware co-simulation approach," International Journal of Electrical Power and Energy Systems, Vol. 81, pp. 346-360, 2016.

[8]. Abdul Gafoor Shaik, Ramana Rao V. Pulipaka, "A new wavelet based fault detection, classification and location in transmission lines,” International Journal of Electrical Power and Energy Systems, Vol. 64, pp. 35-40, 2015.

\section{Biographies}

Neha Agarwal was born in Agra in UP state of India, on Dec. 26, 1989. She studied at Rajasthan College of

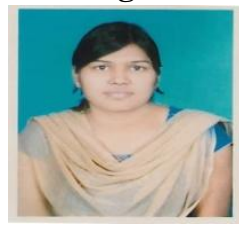
Engineering for Women (RCEW), Jaipur, Rajasthan and received the electrical engineering degree from Rajasthan Technical University (RTU), Kota, Rajasthan in 2011. She is currently pursuing M.Tech (Power System) from Rajasthan Technical University, Kota, India.

Om Prakash Mahela was born in Sabalpura (Kuchaman City) in the Rajasthan, India, on April 11, 1977. He

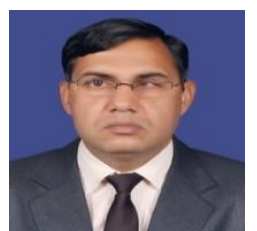
studied at Govt. College of Engineering and Technology (CTAE), Udaipur, and received the electrical engineering degree from Maharana Pratap University of Agriculture and Technology, Udaipur, India in 2002. He is received M.Tech (Power System) from Jagannath University, Jaipur. He is pursuing Ph.D. from Indian Institute of Technology Jodhpur.

His employment experience included the Rajasthan College of Engineering and Technology Jaipur and Rajasthan Rajya Vidhyut Prasaran Nigam Ltd. Presently he is working in the RVPN as Assistant Engineer. His special fields of interest are Transmission and Distribution (T\&D) grid operations, Power Electronics in Power System, Power Quality and Load Forecasting. He is an author of 60 International Journals and Conference papers. Mahela received University Rank certificate from MPUAT, Udaipur, in 2002 and Gold Medal in 2013. He is Student Member of IEEE. He is member of IEEE Communications Society, Power \& Energy Society and Power Electronics Society, He is Fellow of IAEME. He is reviewer of IEEE and Elsevier Journals.

Bipul Kumar received Bachelor of Engineering degree in 2009 with Electrical Engineering from Rajasthan

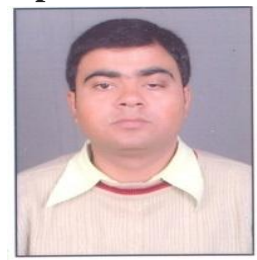
Technical University, Kota, India. He received M.Tech. with power system specialization in 2013 from Jagannath University, Jaipur, India. He is presently working as Assistant Professor with department of Electrical Engineering at Rajasthan college of Engineering for Women, Ajmer Road Bhankrota Jaipur. 